\title{
Supplemental biotin and erroneous thyroid diagnoses and management
}

\author{
Stephanie Charles* and Manfred Blum \\ Department of Internal Medicine, NYU Langone Health, USA
}

Biotin is water-soluble vitamin that acts as a cofactor in enzymatic carboxylation reactions. It plays an important role in the treatment of several inherited metabolic disease (biotinidase deficiency, biotinthiamine-responsive basal ganglia disease), mitochondrial energy disease, and relapsing-remitting multiple sclerosis [1]. Biotin has also gained popularity as an over-the-counter dietary supplement to enhance hair, skin, and nail growth. One of its lesser-known applications is in biotechnology, where it is commonly used in immunoassays to measure various proteins and hormones [2].

Recently, the pediatric literature has described cases of children taking high-doses of biotin who experienced thyroid function test assay interference, resulting in an erroneous diagnosis of Graves' disease [1]. Here we will review several of the documented studies and report on our own findings in the adult population.

Kumer et al [1] described six children with inherited metabolic disease who received high-dose biotin treatment. During routine laboratory evaluation, all six children were found to have lab results suggestive of Graves' disease, with elevated levels of free thyroxine (T4) and total triiodothyronine (T3), low levels of thyrotropin (TSH), and elevated levels of anti-thyrotropin receptor antibodies. Only one child reported hyperthyroid symptoms. Thyroid ultrasound with doppler flow were normal in all patients. Ultimately, the laboratory pattern was attributed to biotin assay interference. After discontinuation of biotin treatment, TSH and thyroid hormone levels normalized within 24-48 hours, while anti-thyrotropin receptor antibodies normalized within 7 days.

Kwok et al. [3] reported on a three-year old girl with the inherited metabolic disease, propionic acidemia, treated with high doses of biotin at $10 \mathrm{mg}$ daily. Baseline thyroid function tests were performed, which revealed low TSH and normal FT4 and T3, indicating hyperthyroidism. However the patient was clinically euthyroid. The authors were suspicious of laboratory interference. They tested the patient's serum with analyzers that utilized biotin versus those that didn't. They discovered that the initial laboratory pattern was erroneously due to biotin interference with the particular TSH assay that employs a biotinstreptavidin interaction.

Pedersen et al. [4] described a case of erroneous biochemical hyperthyroidism in a newborn baby. The mother's first child was diagnosed with biotinidase deficiency during routine neonatal screening, and took $5 \mathrm{mg}$ biotin daily. Therefore, the mother took $10 \mathrm{mg}$ biotin daily from the $20^{\text {th }}$ gestational week of pregnancy until delivery of her second pregnancy. Her newborn baby was found to have thyroid function tests indicative of hyperthyroidism (suppressed TSH and high-normal T4 and T3). The baby was presumed to have neonatal hyperthyroidism, and was hospitalized at 10 days old. However, she appeared healthy and asymptomatic, and a thyroid ultrasound was normal. Ultimately, it became clear to her physicians that her laboratory tests were erroneously due to biotin assay interference, which subsequently normalized as biotin levels were metabolized and excreted.

To understand how this laboratory phenomenon occurs, it is helpful to understand how the biotin-streptavidin interaction works in the standard TSH sandwich immunoassay. The patient's serum is incubated with a biotinylated monoclonal TSH antibody and a ruthenium-linked monoclonal TSH antibody. The serum TSH forms an immune complex with the two antibodies. The immune complex is fixed to the solid phase through the strong interaction between biotin and streptavidin-coated microparticles located on the surface of the well. Voltage is applied to the sample, and the signal produced by the ruthenium is directly proportional to TSH in the serum. Therefore, when there is excess biotin in the patient's serum, fewer TSH-immune complexes will bind to the streptavidin-coated solid phase, and the resulting TSH measurement will be falsely low (Figure 1). In contrast, in assays such as free T4, which involves a competitive process, a falsely elevated level of the hormone will be obtained, further complicating an erroneous diagnosis of hyperthyroidism [5].

In our adult Endocrinology practice, we have encountered this laboratory artifact in patients using much smaller doses of biotin (100300 micrograms) that are found in over-the-counter (OTC) dietary supplements for hair, skin, and nail growth. Therefore, OTC products containing biotin have the ability to cause assay interference and resulting clinical confusion and misdiagnosis. Our findings in adults also highlight that this laboratory artifact adversely affects the diagnosis and management of the entire spectrum of thyroid disorders, not only an erroneous diagnosis of Graves' disease.

In the last year, several prospective trials have been performed to confirm biotin interference in commonly-used TSH assay. Li et al. performed a nonrandomized crossover study of six healthy adults who ingested $10 \mathrm{mg}$ daily of biotin for one week. They measured 11 different analytes, including 9 hormones and 2 nonhormones, using 37 immunoassays (23 of which utilized a biotin-streptavidin system). Specifically, they found that this dose of biotin caused laboratory interference when a biotin-streptavidin system was used to measure serum TSH [6].

Correspondence to: Stephanie Charles, MD, Department of Internal Medicine, NYU Langone Health, New York, NY 10016, USA, E-mail: Stephanie.Charles@nyumc.org

Received: February 22, 2018; Accepted: March 10, 2018; Published: March 13, 2018 


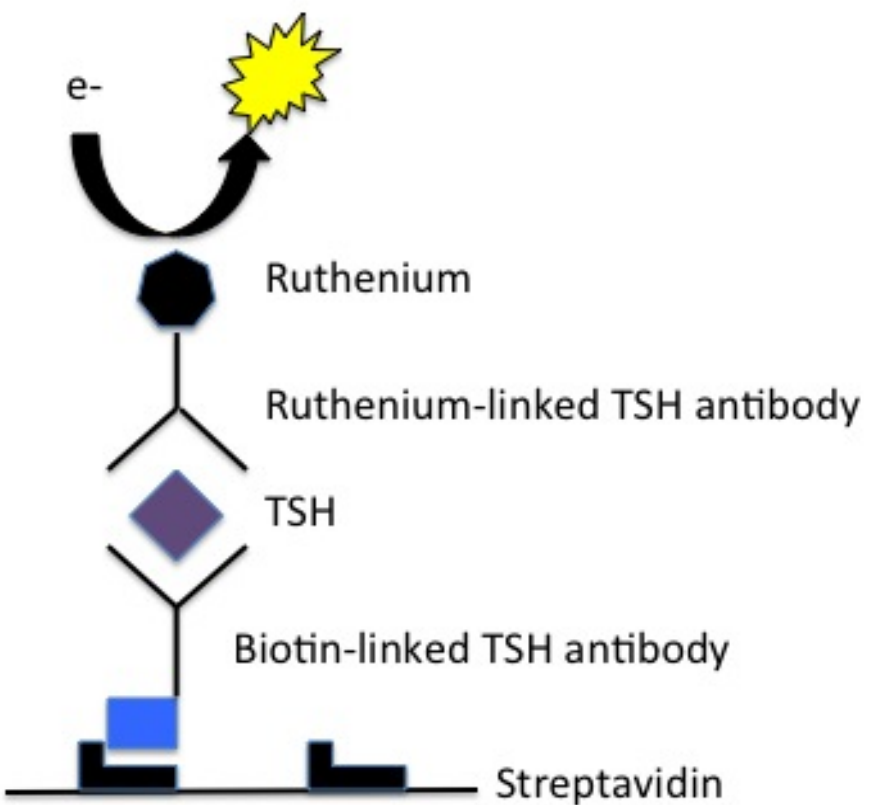

\section{Biotin-streptavidin interaction}

Figure 1. Biotin-streptavidin interaction

We believe it is important to educate clinicians and consumers about this laboratory phenomenon. As large-doses of biotin are indicated in the treatment of inherited metabolic disease in infants and children, it is essential that clinicians who work with these populations be aware of this laboratory artifact. Similarly, TFTs are routinely checked to assess for congenital hypothyroidism, thyroid cancer, and delayed puberty in the pediatric population. However, the population at risk for assay interference is much larger, and includes anyone who uses over-the- counter products containing biotin, including multivitamins. This includes teenagers and adults who use biotin for hair and nail growth, or pregnant young women who use prenatal or multivitamins that may contain biotin.

We recommend that clinicians routinely question their patients about the use of biotin, as a prescribed medication or an over-thecounter supplement. If a patient is taking exogenous biotin, we recommend thyroid function tests be postponed until 24-48 hours after last biotin ingestion.

\section{Acknowledgements}

We have no conflicts of interest to disclose.

Oral presentation: Charles S, Agrawal N, Blum M. Erroneous Thyroid Diagnosis and Management Due to Over-the-counter Biotin. NY Thyroid Club, NY, NY, April 21, 2017.

We extend our thanks to Dr. Barbesino G, for granting us permission to use his graphic in the creative development of our own.

\section{References}

1. Kummer S, Hermsen D, Distelmaier F (2016) Biotin treatment mimicking graves' disease. $N$ Engl J Med 375: 704-706. [Crossref]

2. Diamandis EP, Christopoulos TK (1991) The biotin-(strept)avidin system: principles and applications in biotechnology. Clin Chem 37: 625-636. [Crossref]

3. Kwok JS, Chan IH, Chan MH (2012) Biotin interference on TSH and free thyroid hormone measurement. Pathology 44: 278-280. [Crossref]

4. Pederson IB (2016) Biochemical Hyperthyroidism in a Newborn Baby Caused by Assay Interaction from Biotin Intake. Eur Thyroid J 5: 212-215. [Crossref]

5. Barbesino G (2016) Misdiagnosis of Graves' disease with apparent severe hyperthyroidism in a patient taking biotin megadoses. Thyroid 26: 860-863. [Crossref]

6. Li D, Radulescu A, Shrestha RT, Root M, Karger AB, et al. (2017) Association of Biotin Ingestion With Performance of Hormone and Nonhormone Assays in Healthy Adults. JAMA 318: 1150-1160. [Crossref] 